\section{From Mice to Humans: The Exocrine Pancreas Does Not Matter in Human GLP-1 Receptor Imaging}

TO THE EDITOR: Noninvasive determination of pancreatic $\beta$-cell mass (BCM) in humans is key to understanding the pathophysiology of type 1 diabetes (T1D) and type 2 diabetes. It appears that $\mathrm{BCM}$ and $\beta$-cell function are not directly linked and that $\beta$-cell dysfunction is a key pathophysiologic parameter. The main challenge of pancreatic $\beta$-cell imaging is to use a highly specific tracer molecule so that the signal originating from the pancreas reflects actual BCM (1).

Khera and coworkers show that reduction of exocrine pancreatic uptake by GLP-1 receptor (GLP-1R) blocking with fluorescent exendin improves in vivo imaging of pancreatic $\beta$-cells in mice (2). We have previously demonstrated that, as opposed to mice, rats do not display exocrine pancreatic uptake, as confirmed by quantitative polymerase chain reaction (3) and immunohistochemical GLP-1R staining (4). The human pancreas shows an even higher endocrine-to-exocrine ratio (3), and single-cell RNA sequencing demonstrates specificity of the GLP-1R for human $\beta$ - and $\delta$-cells (5).

Insulin-positive islets are present in pancreata of people with long-standing T1D, indicating that $\beta$-cell dysfunction plays an important role in T1D pathophysiology (6). Pancreatic exendin uptake in individuals with T1D therefore reflects dysfunctional BCM (representing highly important information about the pathophysiology of T1D) and not uptake in non- $\beta$-cells, explaining “ ... the lack of clinical distinction between healthy volunteers and subjects with long-term diabetes ...." as claimed by Khera et al. (2).

We have recently shown in people with long-standing T1D that exendin uptake in human pancreata can indeed drop to background levels, a strong argument against exocrine pancreas uptake. About half the tested individuals showed significant pancreatic uptake, pointing toward residual $\mathrm{BCM}$ (7), in line with the concept of $\beta$-cell dysfunction in T1D (6). Immunohistochemical analysis of human pancreata from individuals with long-standing T1D indeed confirms the presence of numerous GLP-1R-expressing $\beta$-cells and staining of $\delta$-cells, the latter explaining the low residual uptake after complete loss of $\beta$-cells. Data from healthy individuals confirm exclusive staining of $\beta$ - and $\delta$-cells and no staining in exocrine pancreas (7). These data are confirmed by the finding that ex vivo autoradiography of human pancreatic tissue shows only background uptake in the exocrine pancreas (comparable to rats) (8).

Finally, in view of the small differences in exocrine pancreatic uptake between wild-type and GLP-1R knockout mice, shown by Khera and coworkers (Fig. 1) (2), and the minor uptake reduction after blocking (2), the GLP-1R does not play an important role in exocrine pancreas uptake, as shown previously (4).

Therefore, although the approach presented by Khera and coworkers (2) is highly interesting, the practical value is limited to mouse imaging. In addition, high pharmacologic doses of (fluores-

\footnotetext{
(C) 2021 by the Society of Nuclear Medicine and Molecular Imaging.
}

cent) exendin may lead to receptor saturation phenomena disturbing the linear correlation between tracer uptake and $\mathrm{BCM}(1,3)$.

\section{REFERENCES}

1. Eriksson O, Laughlin M, Brom M, et al. In vivo imaging of beta cells with radiotracers: state of the art, prospects and recommendations for development and use. Diabetologia. 2016;59:1340-1349.

2. Khera E, Zhang L, Roberts S, et al. Blocking of glucagonlike peptide-1 receptors in the exocrine pancreas improves specificity for $\beta$-cells in a mouse model of type 1 diabetes. J Nucl Med. 2019;60:1635-1641.

3. Brom M, Woliner-van der Weg W, Joosten L, et al. Non-invasive quantification of the beta cell mass by SPECT with ${ }^{111}$ In-labelled exendin. Diabetologia. 2014;57: 950-959.

4. Willekens SMA, Joosten L, Boerman OC, et al. Strain differences determine the suitability of animal models for noninvasive in vivo beta cell mass determination with radiolabeled exendin. Mol Imaging Biol. 2016;18:705-714.

5. Segerstolpe Å, Palasantza A, Eliasson P, et al. Single-cell transcriptome profiling of human pancreatic islets in health and type 2 diabetes. Cell Metab. 2016;24:593-607.

6. Oram RA, Sims EK, Evans-Molina C. Beta cells in type 1 diabetes: mass and function; sleeping or dead? Diabetologia. 2019;62:567-577.

7. Boss M, Kusmartseva I, Woliner-van der Weg W, et al. In-exendin spect imaging suggests presence of residual beta cells in patients with longstanding type 1 diabetes [abstract]. Diabetologia. 2020;63:S22-S23.

8. Gotthardt M, Jansen TJP, Buitinga M, et al. Validation of exendin for beta cell imaging: ex vivo autoradiography of human pancreas demonstrates specific accumulation of radiolabeled exendin in islets of Langerhans [abstract]. Diabetologia. 2020;63:S179-S180.

\author{
Martin Gotthardt* \\ Lieke Joosten \\ Theodorus J.P. Jansen \\ Maarten Brom \\ Marti Boss \\ Stefanie M.A. Willekens \\ * Radboud University Medical Center \\ Nijmegen, The Netherlands \\ E-mail: martin.gotthardt@radboudumc.nl
}

Published online Feb. 19, 2021.

DOI: 10.2967/jnumed.120.259184

\section{Reply: From Mice to Humans: The Exocrine Pancreas Does Not Matter in Human GLP-1 Receptor Imaging}

REPLY: I want to thank Gotthardt and colleagues for bringing this important clinical question to the forefront of discussion - the independent measurement of $\beta$-cell mass and $\beta$-cell function (1). Indeed, this distinction has major implications for both type 1 and type 2 diabetes research. Their group and others have pioneered glucagon-like peptide-1 receptor (GLP-1R) imaging in the clinic, moving this research beyond preclinical animal models. Despite great progress, significant challenges remain. 